\title{
Nano-Machines on Biological Networks
}

\author{
Arvind Kumar Gupta* and Natasha Sharma \\ Department of Mathematics, Indian Institute of Technology Ropar, India
}

Submission: April 25, 2017; Published:May 25, 2017

*Corresponding author: Arvind Kumar Gupta, Department of Mathematics, Indian Institute of Technology Ropar, India, Tel: +91(01881)242140; Email: akgupta@iitrpr.ac.in

\begin{abstract}
Cells being the underlying entities of living bodies are able to conduct many critical functions. The locally distant cell organelles are connected via metabolic cytoskeleton network, ensuring active and effective intracellular transport. Moreover several kind of mobile biological nano-machines, namely the cytoskeleton motors attach themselves to cytoskeleton filaments and move in opposite direction to transport cargo vesicles from one particular location to another. In this mini review we attempt to elucidate how unlike motors and motor teams coordinate to contribute in efficient unidirectional and bidirectional transport, including in specific, the significance of motor-motor interactions and the transformations of the underlying transport network. Our short review may provide a framework to perceive intracellular transport operation in cell organelles.
\end{abstract}

Keywords: Molecular motors; Cytoskeleton; Intracellular transport; Microtubules; Dynein; Kinesin

\section{Introduction}

The cell being the building block of life is the fundamental unit of structure and function in all living organisms. The cell organelles are equipped for carrying out one or more vital tasks. These operations depend on progressive microscopic processes, directed by steady energy supply and are robustly out-of-equilibrium systems. In order to sustain the cell functionality, active transportation of various vital ingredients is required at diverse intracellular locations. Due to cell crowding and lengthening at micro levels, these operations cannot be accomplished by diffusion only. These organelles are attached to the cytoskeleton network consisting of protein microfilaments, intermediate filaments, and microtubules. Intracellular cargos such as lipid droplets, endosomes, mitochondria, and various proteins and viruses are periodically transported many micrometers along these well-defined routes and are delivered to particular addresses [1]. Microfilaments, composed of linear polymers of G-actin proteins, act as tracks for the movement of myosin molecules that attach to the microfilament and walk along them. Intermediate filaments are heterogeneous constituents of the cytoskeleton and function in the maintenance of cell-shape. Among these filaments, microtubules (MTs) are the stiffest cytoskeleton filaments having the most convoluted association and disassociation progression. MTs are polymers with fundamental subunits, $\alpha / \beta$ - tubulin of $8 \mathrm{~nm}$ length, that spontaneously gather along their long axis into protofilaments of approximately $25 \mathrm{~nm}$ of diameter. Generally, twelve to fourteen of these protofilaments enfold into a helical cylinder known as a MT, which serves as a track for long distance intracellular transport carried out by nano molecular motors of the kinesin and dynein family. The dissimilarity of the dimer subunit results in the polar nature of the MTs. The filament bound with $\beta$ - tubulin charaterize the plus-end of the MT and the $\alpha$-tubulin tip is the negative terminal [2,3]. However, the MT lattice displays a twist and, in addition, a small preferred curvature due to the internal organization of the protofilaments. Owing to the helical nature of MTs, both circular and wavy trajectories have been observed experimentally, indicating that the protofilaments assume curved conformations [4]. In addition, they are highly dynamic structures that continually associate and disassociate through the addition and removal of tubulin heterodimers at their terminal. During the formation of the MT filament, free $\alpha$ - and $\beta$-tubulin binds to the GTP (guanosine triphosphate) nucleotide. After polymerization, the GTP bound to the $\beta$ - tubulin hydrolyzes to GDP (guanosine diphosphate). Hence, while the microtubule filament is constructed from GDP-tubulin, there is a cover of GTP bound $\beta$ - tubulin at the lately polymerized microtubule plus tip. Due to its higher sustainability, the GTP-bound microtubule tip prevents microtubule disassembly. As soon as the stabilizing tip is lost, the microtubule shifts to the depolymerizing phase (called catastrophe), whereas recovering the GTP plus tip is associated with diverting back to growth (called rescue). Both plus and minus tips of a microtubule display dynamic instability, 
but they differ in the frequencies of catastrophe and rescue [5]. In addition, recent biochemical experiments have proved that specific proteins also alter the length dynamics of MTs. Kip3p, a molecular motor of the kinesin-8 protein family is an MTplus end depolymerizing agent, where as XMAP215 is a protein that amplifies it's growth rate [6-8]. Further $\gamma$-TURC, CAMSAP, Patronin, Nezha family proteins greatly facilitate the analysis of microtubule minus-end dynamics [5]. From an extensive opinion, it is clear that unlike the roads of human assembly, microtubules are very dynamic and sensitive. This type of observation may serve as inquisitiveness for understanding how special kind of carrier, namely molecular motors travels on these dynamic and unstable roads. More generally, how a system of bidirectional motors can function together and differently in a variety of situations. In the following section we review alternative scenarios which lead to efficient bidirectional active transport and regulation of cellular cargo by avoiding jamming.

\section{Discussion}

Motor proteins, known as cytoskeleton motors, are molecules which are capable of moving along polar cytoskeleton filaments such as MTs or actin filaments. The potential acquired from hydrolysis of adenosine triphosphate (ATP) encourage a configurational change of the motor protein resulting into forward stepping along the filament. The motor domain component of the protein binds to the filament, while the cargo domain binds to intracellular cargos or other proteins of the cytoskeleton. Most kinesins motors proceed to the MT-plus end (away from the nucleus) whereas dynein progress towards MTminus end (toward the nucleus) while carrying cargos [9]. Local transport occurs on actin filaments through unconventional myosin proteins in regions where there are less microtubule. Quite recently, however, kinesin-5 Cin8 and kinesin-14 KlpA is found to exhibit directionality switching, depending on the number of molecular motors bound to the same MT [10-11].

Kinesins are $80 \mathrm{~nm}$ long motors and have two globular heads with diameters of $10 \mathrm{~nm}$ each. During the progression it takes an $8 \mathrm{~nm}$ long step and is capable to exert a stall force up to 6 $\mathrm{pN}$ to the cargo [12]. Dyneins bind to the microtubule with two binding stalks, $13 \mathrm{~nm}$ long, exerting force of about $1.1 \mathrm{pN}$ and are nearly 10 times larger than kinesins [13]. The step size of dynein varies within $8,18,24$ and $32 \mathrm{~nm}$. Further along with forward hopping molecular motors can detach and attach to the filaments as well. Vitro experiments have revealed that kinesins carry about 100 steps before separating from the MT and after a short time, they associate again. This scenario reoccurs over again till the load reaches the target. Since the total steps before unfixing are relatively high, kinesins are also called processive motors. The portion of the percentage time for which motors are fixed to the filament during the progression is attributed as the duty ratio. Kinesins have a duty ratio of almost one, indicating that the instance between the detachment and reattachment is radically small. Dyneins, on the other hand have a notably lower processivity [9].

In some systems, the same load extends on both microtubule and actin filaments, accompanied by multiple molecular motors in the route of passage. Though a single motor protein can accomplish processive motion, convey in cells is frequently carried by collective motors. One benefit of load carried by unidirectional bunch of several motors is an increased run length compared with the run length of an individual motor. Further, if one motor unbinds, the cargo is still transferred by the other motors and the detached motor has a chance to reattach to the filament. In this way, load can be conveyed over typical cellular distances [14]. Further multiple, oppositely directed motors simultaneously perform and encounter each other on a given microtubule. An operational co-ordination seems to exist between MT and actin filament webs, and within variety of motors for efficient transport network. A harmonized interchanging of motor activity and tug-of-war (TOW) scenarios has been considered to explain the interactions between opposite motors $[14,15]$. In the switching model, in a mutually exclusive manner foreign non motor regulatory proteins activate/inactivate the appropriate motors associated with the load. As a result, conflicting motors never produce resistant against each other. In disparity, TOW postulate that opposite motors actually tries to pull the load complex in its particular direction against each other. Kinesins haul towards the plus end, and dyneins drag towards the minus end. One with larger strength detaches the other. Other alternatives comprehend that due to poor processivity and propensity to back-step, dynein clear the way to oppositely moving motor protein. Further, it can re-associate to adjacent protofilaments of the microtubule in due course of progression. Moreover, experiments have revealed that Kinesin-1, particular member of kinesin family, on other hand, due to its high processivity proceed on single filament and override dynein-propel load to an adjacent protofilament [16]. All these possible scenarios results in smooth bidirectional transport. In another intuitive situation, to switch within MT and actin filament, actin motors as well as microtubule motors hook up simultaneously on the same cargo. Switching between variant filament entities depends on the load and on the number and type of molecular motors [14]. The proper operation of motor transport is crucial for the cell's survival, since, its disturbance can contribute to the evolution of diseases such as Alzheimer's, hearing loss, virus transport, and neurodegenerative and polycystic kidney diseases [17]. In addition to in in vitro and in vivo experiments to understand the function of molecular motors, the exact cycle of events are extensively studied by an effective model based on the totally asymmetric exclusion process- TASEP. TASEP and its variants has also been used to confront the effective interaction with the filament environment, either because of forward stepping or vital convey across nearby filaments; and through particle attachment or detachment, both 
in unidirectional and bidirectional transport [18-21]. These models are helpful to enhance our qualitative insight into the complex dynamics of motor protein transport networks.

\section{Conclusion}

We have discussed variant modes of co-operative transfer: unidirectional transfer, bidirectional transfer and transfer on varied tracks. The regular functioning of cytoskeleton transport is critical for the endurance of the cell, since, its mutation and disruption can results in fatal diseases. In this direction, our short review tenders to a significant understanding of cargo transport by distinct molecular motors along filament networks. To some extent we viewed how several molecular motors interact and co-operate at micro level to ensure smooth transport of vital cargoes, for proper functioning and maintenance of cellular activities.

\section{References}

1. Lodish H (2008) Molecular cell biology. In: Lodish H (Ed.), Molecular cell biology ( $8^{\text {th }}$ edn), Macmillan, p.1280.

2. Schaletzky J, Rape M (2016) Getting a Grip on Microtubules. Cell 164(5): 836-837.

3. Schliwa M, Woehlke G (2003) Molecular motors. Nature 422(6933): 759-765.

4. Gosselin P, Mohrbach H, Kulić IM, Ziebert F (2016) On complex, curved trajectories in microtubule gliding. Physica D Nonlinear Phenomena 318: 105-111.

5. Akhmanova A, Hoogenraad CC (2015) Microtubule minus-endtargeting proteins. Curr Biol 25(4): R162-R171.

6. Reese L, Melbinger A, Frey E (2014) Molecular mechanisms for microtubule length regulation by kinesin-8 and XMAP215 proteins. Interface focus 4(6): 20140031.

7. Varga V, Leduc C, Bormuth V, Diez S, Howard J (2009) Kinesin-8 motors act cooperatively to mediate length-dependent microtubule depolymerization. Cell 138(6): 1174-1183.

8. Reese L, Melbinger A, Frey E (2011) Crowding of molecular motors determines microtubule depolymerization. Biophys J 101(9): 21902200 .
9. Appert-Rolland C, Ebbinghaus M, Santen L (2015) Intracellular transport driven by cytoskeletal motors: General mechanisms and defects. Physics Reports 593: 1-59.

10. Saito N, Kaneko K (2017) Embedding dual function into molecular motors through collective motion. Scientific Reports 7, doi:10.1038/ srep44288.

11. Popchock AR, Tseng KF, Wang P, Karplus PA, Xiang X, et al. (2017) The mitotic kinesin-14 KlpA contains a context-dependent directionality switch. Nat Commun 8: 13999.

12. Furuta KY, Furuta A, Toyoshima YY, Amino M, Oiwa K, et al. (2013) Measuring collective transport by defined numbers of processive and nonprocessive kinesin motors. Proc Natl Acad Sci 110(2): 501-506.

13. Bhabha G, Johnson GT, Schroeder CM, Vale RD (2016) How dynein moves along microtubules. Trends Biochem Sci 41(1): 94-105.

14. Berger F, Keller C, Mueller MJ, Klumpp S, Lipowsky R (2011) Cooperative transport by molecular motors. Biochem Soc Trans 39(5): 1211-1215.

15. Soppina V, Rai AK, Ramaiya AJ, Barak P, Mallik R (2009) Tug-of-war between dissimilar teams of microtubule motors regulates transport and fission of endosomes. Proceedings of the National Academy of Sciences 106(46): 19381-19386.

16. Mallik R, Gross SP (2004) Molecular motors: strategies to get along. Curr Biol 14(22): R971-R982.

17. Gupta AK (2016) Collective dynamics on a two-lane asymmetrically coupled TASEP with mutually interactive Langmuir Kinetics. Journal of Statistical Physics 162(6): 1571-1586.

18. Kuan HS, Betterton MD (2016) Motor protein accumulation on antiparallel microtubule overlaps. Biophysical journal 110(9): 20342043.

19. Sharma N, Gupta AK (2017) Phase segregation and spontaneous symmetry breaking in a bidirectional two-channel non-conserving model with narrow entrances. J Stat Mech 2017.

20. MacDonald CT, Gibbs JH, Pipkin AC (1968) Kinetics of biopolymerization on nucleic acid templates. Biopolymers 6(1): 1-5.

21. Melbinger A, Reese L, Frey E (2012) Microtubule length regulation by molecular motors. Physical review letters 108(25): 258104.

\section{Your next submission with Juniper Publishers} will reach you the below assets

- Quality Editorial service

- Swift Peer Review

- Reprints availability

- E-prints Service

- Manuscript Podcast for convenient understanding

- Global attainment for your research

- Manuscript accessibility in different formats

( Pdf, E-pub, Full Text, Audio)

- Unceasing customer service

Track the below URL for one-step submission https://juniperpublishers.com/online-submission.php 